\title{
The effect of an indoor-outdoor temperature difference on transient cross-ventilation
}

\author{
M. S. Davies Wykes ${ }^{\mathrm{a}, *}$, E. Chahour ${ }^{\mathrm{b}}$, P. F. Linden ${ }^{\mathrm{c}}$ \\ ${ }^{a}$ Engineering Department, Trumpington Street, Cambridge, UK, CB2 1PZ \\ ${ }^{b}$ INSA Lyon, 20 Albert Einstein Ave, 69621 Villeurbanne Cedex, France \\ ${ }^{c}$ DAMTP, Centre for Mathematical Sciences, Wilberforce Road, Cambridge, UK, CB3 OWA
}

\begin{abstract}
We examine the effect of an indoor-outdoor temperature difference on the transient wind-driven cross-ventilation of a room. Laboratory experiments are performed in a water flume using a reduced-scale model room. For solely winddriven cross-ventilation with no initial temperature difference between the room and the external fluid, the ventilation rate is constant. In experiments, the mean dye concentration decays exponentially, which is expected when the room remains well-mixed. When there is an initial temperature difference but no wind, the buoyancy-driven exchange-ventilation results lie between a model that assumes the room is well-mixed and a new model that assumes no mixing between the incoming flow and the room. When both wind and buoyancy drive the flow, the relative importance of these two effects can be described by a Froude number, $F r$. For buoyancy-dominated ventilation $(F r<1)$, the ventilation rate through the windows can be modelled using an exchange flow with a $F r$ correction to account for wind effects, which can increase the ventilation rate by up to $40 \%$. For wind-dominated ventilation $(F r>1)$, a temperature difference slightly reduces the ventilation rate, but only by up to $6 \%$, a change that can be neglected in most applications. Two processes compete to ventilate the room in combined cases: the removal of fluid from a lower layer by flow through the windows and the erosion of an upper layer by entrainment into the jet that crosses the room. The relative rates of these two processes depend on the geometry of the room.
\end{abstract}

Keywords: natural ventilation; cross-ventilation; wind;

\footnotetext{
* Corresponding author

Email address: msd38@cam.ac.uk (M. S. Davies Wykes)
} 


\section{Introduction}

Heating and cooling account for a significant proportion of energy use in buildings (e.g. $50 \%$ in the USA [1]). These high energy costs could be reduced by using ventilation strategies that make use of naturally occurring wind and buoyancy forces to drive flows. However, people have become accustomed to well-controlled indoor conditions, which are difficult to achieve when ventilation is strongly affected by outdoor conditions. Increased uptake of natural ventilation will require simple, accurate models that can be integrated into building design and control systems. In this paper, we examine the effect of an initial temperature difference on the transient wind-driven cross-ventilation of a room, using the results of laboratory experiments to build mathematical models that describe the flow.

There has been a wealth of research into natural ventilation $[2,3]$. We are principally concerned here with buoyancy-driven flows through relatively large openings, where there can be an exchange flow through an opening [4]. Early work on buoyancy-driven exchange flows through an opening in a vertical wall was carried out by Brown and Solvason [5]. Phillips and Woods examined the transient ventilation of a room through a doorway using laboratory experiments [6]. They found that a two-layer stratification formed in the room, where the interface between the layers was well described by a model that assumed fluid flowing into the room through the doorway did not mix with the fluid in the room and that the flow was hydraulically controlled at the doorway. Full scale experiments of transient buoyancy-driven exchange ventilation were carried out by Heiselberg and Perino, who identified two stages of ventilation: short-term displacement ventilation followed by slower mixing ventilation [7].

Previous research on the interaction between wind and temperature for natural ventilation has concentrated on the effect of wind on stack-driven displacement ventilation [see, e.g. 8, 9, 10, 11, 12, 13, 14, 15, among others], where ventilation between high and low openings is driven by a temperature difference between the building and the surrounding environment. For stack-driven ventilation, the heights of the openings are generally small compared to the difference in height between openings. In contrast, for cross-ventilated rooms, the height difference between the openings is small compared to the heights of the openings. An investigation of the effect of temperature on cross-ventilation was performed by Stavridou et al., although the experiments performed in that study had a height difference between openings so that there was a significant stack-driven component to the flow [16]. In this paper we will examine the case where the openings are at the same height.

Research has also been conducted that examines the interaction of wind with 
single-sided ventilation. Larsen and Heiselberg discovered that the dominating effect on the ventilation rate switched between wind and temperature, depending on the ratio of the relevant forces [17]. Heiselberg et al undertook full-scale experiments of forced unidirectional flow through a side-hung window and found the effective discharge coefficient decreased for large temperature differences, suggesting a transition from wind-dominated to buoyancy-dominated conditions [18].

The effect of a buoyancy source on the cross-ventilation of a building with multiple rooms has been examined by Lo and Novoselac, using full-scale experiments and CFD simulations [19]. They found that the buoyancy source (located outside of the path of the cross-ventilation flow) enhanced mixing between different rooms, making cross-ventilation more effective. Lo and Novoselac concentrated on cases where the flow was steady and dominated by cross-ventilation and did not examine the effect of buoyancy on the ventilation rate.

Research has also examined the interaction between a forced net flow rate through an opening with a buoyancy-driven exchange flow. Early research into the interaction between a buoyancy-driven exchange flow through an opening and a forced net flow rate was carried out by Shaw [20]. Shaw developed a theory for the steady case, where he related the forced net flow rate to a pressure difference across the opening. Additional work by Epstein and Kenton examined the effect of combined forced and buoyancy-driven exchange flow through an opening in a horizontal partition [21, 22]. They developed a separate theory for the case where there is an imposed net volume flux through an opening.

In this paper we examine the interaction of an imposed flow velocity across an opening through which buoyancy driven exchange ventilation is taking place. In our example this interaction determines the ventilation rate, rather than there being an imposed ventilation rate through the opening. The models we will develop are related to those developed by Shaw, as the imposed flow results in a dynamic pressure difference across the room. Unlike Shaw, we examine the transient case, where ventilation will change the internal conditions in the room, which then affect the ventilation rate.

The case we have examined is the transient ventilation of a room that is ventilated by a combination of wind-driven cross-ventilation and an initial temperature difference between the inside and outside of the room. The wind-driven cross-ventilation is through two identical windows located at the same positions, one on the windward face and one on the leeward face of the room, as sketched in figure 1. We find that the transient nature of the flow has significant impacts on the evolution of the stratification in the room, which then feeds back into the net ventilation rate of the room with time.

We describe the experimental setup in $\S 2$ and discuss the validity of small 


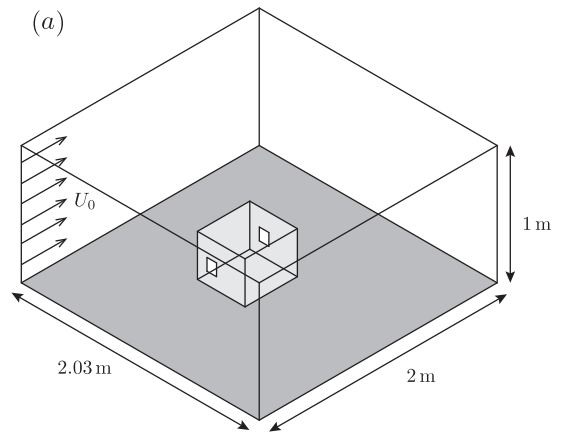

(b)

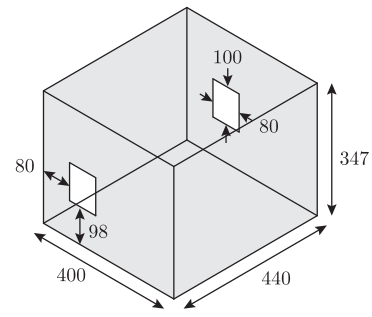

Figure 1: Sketch of (a) the working section of flume with the model room (to scale), and (b) the model room with internal dimensions in $\mathrm{mm}$. Note that the model room has double-glazed walls that are $30 \mathrm{~mm}$ thick, which are not shown.

scale models in $\S 3$. We then describe the results, beginning with wind-driven cross-ventilation in $\S 4.1$, moving on to buoyancy-driven exchange ventilation in $\S 4.2$, then describing the effect of both wind and buoyancy in $\S 4.3$. The late-time behaviour is discussed in $\S 5$ and our conclusions are presented in $\S 6$.

\section{Experimental setup}

The experiments were conducted in a flume that is $2.03 \mathrm{~m}$ wide and filled to a depth of $0.825 \mathrm{~m}$. The working section of the flume is $2 \mathrm{~m}$ long, which is viewable through a $2 \mathrm{~m} \times 1 \mathrm{~m}$ window (see figure $1(a)$ ). The flow speed in the flume at full-width is a maximum of $40 \mathrm{~mm} \mathrm{~s}^{-1}$. The model room with internal dimensions $400 \mathrm{~mm}$ wide, $347 \mathrm{~mm}$ high and $400 \mathrm{~mm}$ long, has a heated floor that was used to create an initial temperature difference between the fluid in the model room and the ambient fluid in the flume. The heated floor was only used before the beginning of an experiment to create the initial temperature difference and was switched off before an experiment began. In order to minimise heat losses from the model room the walls are double-glazed and insulated with air gaps.

The model room was placed centrally in the flume, raised by $100 \mathrm{~mm}$ (above the height of the boundary layer which was $<50 \mathrm{~mm}$ as measured using dye), oriented such that the oncoming flow was parallel to its long dimension. Measurements of the ambient flow using dye streaks showed that the oncoming flow was laminar and uniform over the width and depth of the flume corresponding to the front façade of the model room. The 'windows' were identical rectangular openings $100 \mathrm{~mm}$ high and $80 \mathrm{~mm}$ wide, with the bottom edge set $98 \mathrm{~mm}$ from 
the floor of the room and located exactly opposite each other on the windward and leeward façades which were normal to the external flow (see Fig. 1(b)). Both openings were initially blocked with dense sponges that were removed at the start of an experiment. The removal of the sponges perturbed the flow by generating a vortex ring inside the model room with the removal of the second sponge, but this perturbation died away within the first 10 seconds and is not considered to significantly impact the ventilation flow over longer time-scales. The experiments typically took 20-30 minutes.

The experiment was backlit using a projector at a distance $6 \mathrm{~m}$ from the flume wall (this distance was achieved by use of two $45^{\circ}$ mirrors). Nearly collimated light passed through the experiment and was projected onto a sheet of tracing paper on the near-side of the flume (the side with the camera). Temperature changes in the fluid lead to variations in the refractive index. The refractive index variations result in patterns of light and dark on the projected image of the experiment, highlighting regions with high density gradients, a technique known as shadowgraph [23]. This gives a qualitative view of the flow.

Temperature was measured using 14 thermocouples: 10 in a vertical array in the centre of the model room (30 $\mathrm{mm}$ apart, with the lowest $30 \mathrm{~mm}$ from the floor) and 4 in the flume to measure the ambient temperature (2 upstream and 2 downstream of the model room). Data was acquired from the thermocouples using Matlab, a National Instruments USB CompactDAQ Chassis and thermocouple input module (NI cDAQ-9171 and NI 9213). The thermocouples were hermetically sealed T-type (Omega HSTC-TT-TI-24S). We measured the timeconstant of the thermocouples to be $<3$ seconds, which is short compared the timescale of temperature changes in the experiments. The thermocouples had low noise, as measured by placing the thermistors in a constant temperature water bath, the standard deviation of the signal was $<0.01 \mathrm{~K}$. The thermocouples were calibrated relative to an alcohol thermometer to remove an offset that varied with temperature and position in the input module. Thirty-three temperature samples were used to calibrate the thermocouples. As we are primarily concerned with differences in temperature between thermistors, the error was characterised using the difference between temperature measured by each of the 14 thermistors and the mean of all thermistors for each of the calibration measurements. The standard deviation of this difference is $0.032 \mathrm{~K}$. Error bars in figures $5 \mathrm{~b}, 8 \mathrm{~b}, 11 \mathrm{~b}$, and 12a are twice this standard deviation.

The heat loss from the insulated model room is discussed in detail in appendix Appendix B. We can compare the rate of heat loss through the walls of the room with the rate of heat loss due to ventilation. The heat lost through the walls will be less than $10 \%$ of the heat loss by buoyancy-driven ventilation so long as the temperature difference $\Delta T>0.02 \mathrm{~K}$. Heat loss through the walls 
results in an exponential decay in temperature with a timescale $\tau_{\text {cool }}$, whereas wind-driven ventilation results in an exponential decay with a timescale $\tau_{\text {wind }}$ (as will be explained in $\S 4.1$, equation 3 ). The ratio of these timescales for the slowest speed $U=18 \mathrm{~mm} \mathrm{~s}^{-1}$ is $\tau_{\text {cool }} / \tau_{\text {wind }}=70$. As heat loss occurs over a much longer timescale than the ventilation processes we are interested in, we will neglect the heat loss from the room.

A small amount of red food dye was added to the fluid in the model room before the start of the experiment. The dye attenuates the light that passes through the room. The attenuation of light can be related to the dye concentration $c$ by use of the Lambert-Beer law,

$$
\frac{I}{I_{0}}=e^{f(c) b},
$$

where $I$ is the light intensity, $I_{0}$ is the intensity of a background image with no dye, $b$ is the distance travelled through the solution, and $f(c)$ is a function of concentration $[24,25]$. We calibrate $f(c)$ using known dye concentrations. To improve the dynamic range of the dye measurements, a green filter was used on the camera. Typical concentrations of red food dye used in experiments were less than $25 \mathrm{ml}$ of $1 \%$ solution in the model room $\left(c<4 \times 10^{-6} \%\right.$ by volume). The dye has a neglible change $\left(<10^{-4} \%\right)$ in the density of the fluid in the model room.

To identify the effect of the shadowgraph on the dye attenuation, an experiment was performed with no dye, with an initial temperature difference of $4 \mathrm{~K}$ and no imposed flow. We found that the light intensity, horizontally averaged across the room, varied by as much as $15 \%$. For this reason, dye attenuation measurements are only used as a quantitative measurement for the wind-only cross-ventilation experiments. For the experiments that have an initial temperature difference between the inside and outside of the model room, the mean temperature of a vertical array of thermocouples was used to characterise the ventilation rate.

Results are presented below from a total of 20 experiments covering a range of 'wind' speeds $U_{0}$ from $0-35 \mathrm{~mm} \mathrm{~s}^{-1}$ and initial temperature differences $\Delta T_{0}$ from $0-16.3 \mathrm{~K}$, giving a range of initial Froude numbers $F r_{0}$ (defined in (17) below) from $0.35-1.72$. A list of the experiments is given in Appendix A. Images from experiments $4,8,11$, and 18 have been used to illustrate the different ventilation cases.

\section{Scaling considerations}

Although small-scale modelling using a water bath technique is a well established method, most previous ventilation research using this method has used 
salt solutions to generate density differences. When using salt solutions, we can achieve dynamic similarity between laboratory experiments and full-scale buildings. This is measured by the Reynolds number $R e \equiv U L / \nu$ of the flow, where $U$ and $L$ are characteristic velocity and length scales and $\nu$ is the kinematic viscosity of the fluid. The Reynolds number provides a measure of the inertia of the flow to frictional forces and for small scale models with reduced length scales there is a potential for frictional effects to become dis-proportionally high. One effect of using heat is that the density differences reachable by our experiment are smaller than they would be if we used salt, thereby reducing $U$ when the flow is buoyancy-driven and potentially affecting the Reynolds number. Another potentially important difference is that the diffusion of temperature occurs at a faster rate than it would for salt, affecting the Péclet number $P e \equiv U L / \kappa$, where $\kappa$ is the molecular diffusivity of the stratifying agent (heat in the case of a real building). A detailed discussion of the validity of using small-scale thermally-driven water bath experiments is given in Partridge et al. [26]. A more general discussion of similarity effects in ventilation experiments is given by Etheridge [27].

Running reduced-scale experiments impacts the Reynolds number of winddriven ventilation and the buoyancy-driven ventilation in different ways. As we are primarily interested in the competing effects of wind and buoyancy on the flow through openings, we will use the window height $h$ as our relevant length scale. For wind-driven flow, if we calculate a full-scale Reynolds number based on a window height $h=0.5 \mathrm{~m}$, wind velocity $U=1 \mathrm{~m} \mathrm{~s}^{-1}$, and the kinematic viscosity of air $\nu_{\text {air }}=1.5 \times 10^{-5} \mathrm{~m} \mathrm{~s}^{-2}$, we find $R e=U h / \nu_{\text {air }}=3.3 \times 10^{4}$. In our experiments, the Reynolds number based on these parameters is smaller: for the slowest 'wind' speed $U=18 \mathrm{~mm} \mathrm{~s}^{-1}$, the $R e=1.8 \times 10^{3}$, based on the window height of $100 \mathrm{~mm}$ and the kinematic viscosity of water $\nu_{\text {water }}=1.0 \times 10^{-6} \mathrm{~m} \mathrm{~s}^{-2}$. However, this is significantly larger than the critical opening Reynolds number $R e=300$ for which flow through a small square window into a room in the form of a jet becomes fully turbulent [28], and so we expect that the flow through the window and inside the room, when appropriately scaled, to be a quantitatively accurate when applied to full scale.

The Reynolds number based on building height ranges from $6 \times 10^{3}$ to $1.3 \times$ $10^{4}$. Studies suggest that a Reynolds number of $2 \times 10^{4}$ is required for Reynolds number independence of turbulent flow around a bluff body. We will therefore not be correctly resolving the effects of a turbulent flow around the building on the cross-ventilation. Turbulence can have significant effects on ventilation [29]. In this study we are examining the competition between temperature and wind, future studies will be needed to examine the interaction of these two effects with turbulence around the building. Note that we have not attempted to generate 
a turbulent boundary layer over the model room.

For the buoyancy-driven flow, we can calculate a Reynolds number as $R e=$ $h \sqrt{h g \gamma \Delta T} / \nu$, where $\Delta T$ is a temperature difference, $\gamma$ is the thermal expansion coefficient, and $g=9.81 \mathrm{~m} \mathrm{~s}^{-2}$ is acceleration due to gravity. At full scale, for a temperature difference $\Delta T=5 \mathrm{~K}$, and $\gamma_{a i r}=3.43 \times 10^{-3} \mathrm{~K}^{-1}$, we find a $R e=1.0 \times 10^{4}$. In our model room, the smallest temperature difference used was $\Delta T=1 \mathrm{~K}$, and $\gamma_{\text {water }}=0.207 \times 10^{-3} \mathrm{~K}^{-1}$, we find $R e=1.4 \times 10^{3}$, which is an order of magnitude smaller. However, this Reynolds number is sufficiently high to expect separation to occur at the edges of the opening, just as for unidirectional flow.

The small scale experiments of Kiel found that the discharge coefficient (defined at the beginning of $\S 4.1), C_{d}=0.6$ for $1 \times 10^{4}<R e<15 \times 10^{4}$, suggesting that the flow is Reynolds number independent over this range of Reynolds numbers [30]. We also find in $\S 4.1$ that $C_{d}=0.6$ suggesting that this result applies for even lower values of Re. In contrast, the full-scale experiments of Wilson and Kiel found $C_{d}=0.4-0.6$ for $1 \times 10^{4}<R e<20 \times 10^{4}$ [31]. This difference was ascribed by the authors to interfacial mixing between the incoming and outgoing streams, likely induced by ambient turbulence in the flow. In the experimental setup used for this study, turbulence levels are low, therefore we expect the flow to follow the results of Kiel [30]. Turbulence can have a significant effect on the flow, which is an important area for future study.

In full-scale buildings, heat transport is dominated by advection over diffusion. This is characterised by a high Péclet number, where $P e=h \sqrt{h g \gamma \Delta T} / \kappa$, where $\kappa$ is the molecular diffusion coefficient. At full scale, with the same temperature difference and window height and using $\kappa_{\text {air }}=2.1 \times 10^{-5} \mathrm{~m} \mathrm{~s}^{-2}$, we find $P e=6.8 \times 10^{3}$. In our experiments, $\kappa_{\text {water }}=1.4 \times 10^{-7} \mathrm{~m} \mathrm{~s}^{-2}$, therefore our $P e=1.0 \times 10^{4}$. These calculations show that, despite the increase in thermal conductivity of water as compared to air, the Péclet number in our experiments matches the full scale.

\section{Results and Discussion}

We will begin by comparing the wind-driven and buoyancy-driven ventilation cases to established models, before moving on to the combined effects of wind and buoyancy.

\subsection{Wind-driven cross ventilation}

If the ventilation is solely wind-driven, the flow rate through the windward opening will be $Q=A^{*} U_{0}$, where $U_{0}$ is the wind speed, and $A^{*}$ is an effective window area. If the windows are identical with equal areas $A$, then $A^{*}=$ 

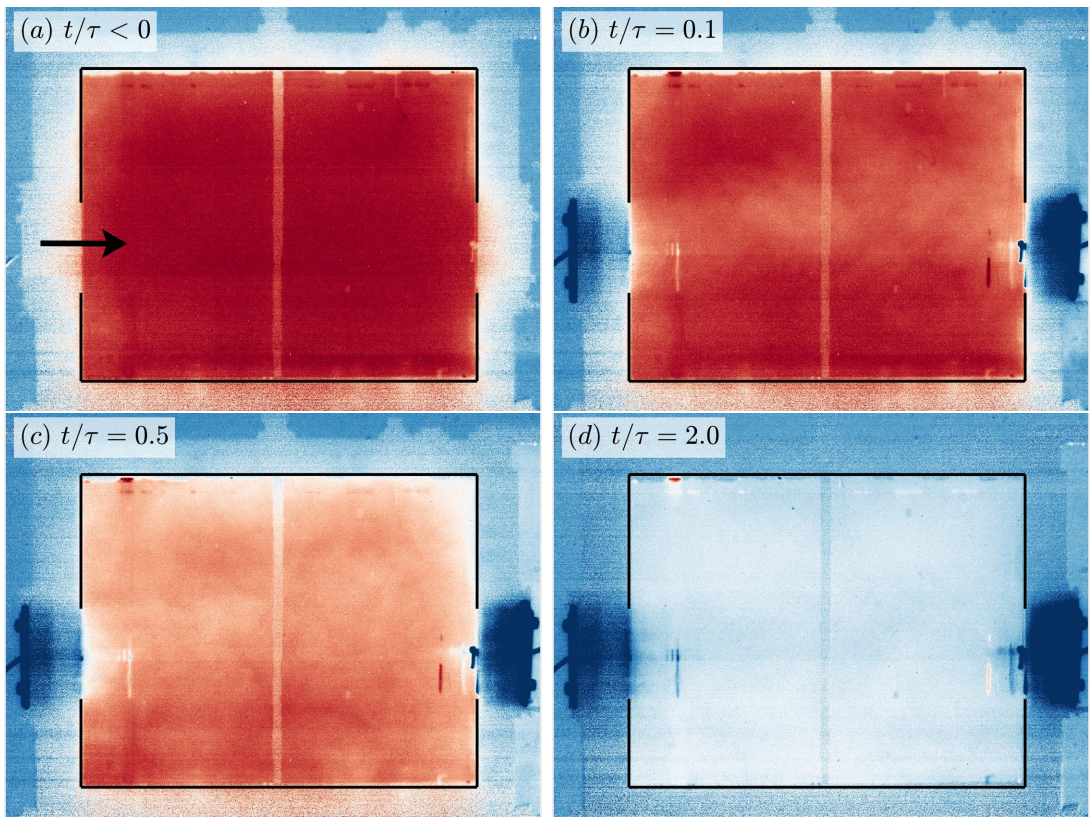

Figure 2: Wind-driven ventilation: images showing the model room (a) before the start of an experiment $\left(t / \tau_{\text {wind }}<0\right)$, (b) $t / \tau_{\text {wind }}=0.1$, (c) $t / \tau_{\text {wind }}=0.5$, (d) $t / \tau_{\text {wind }}=2.0$, where $\tau_{\text {wind }}=V / A^{*} U_{0}$. The images are from experiment 4 from table A.1. The external flow is from left to right, as indicated by the arrow in $(a)$. False colour corresponds to intensity normalised by background intensity. Red corresponds to dyed fluid, while blue is undyed. The interior boundaries of the room and the windows are indicated with overlaid lines. 
$C_{d} A$. The constant $C_{d}$ is an empirical discharge coefficient that accounts for pressure losses and streamline contraction at the window. The value of $C_{d}$ varies depending on the geometry of the opening, with $C_{d}=0.6$ the generally accepted value for a square-edged opening where the ratio of window area to wall area is small [32]. This value has been measured for natural ventilation of fullscale buildings [33]. If the windows have different areas or pressure losses, then $A^{*}=A_{1} A_{2} / \sqrt{\left(A_{1}^{2} / C_{1}^{2}+A_{2}^{2} / C_{2}^{2}\right) / 2}$, where $A_{1}, A_{2}$ are window areas and $C_{1}, C_{2}$, are the discharge coefficients associated with the loss in pressure (dissipation) at each window [9].

It is well known (see e.g. [34], pp. 264) that if the room is well-mixed, for a constant wind speed, and therefore a constant $Q$, the decay rate of the volume average concentration $\bar{c}$ of a passive tracer in the room will be exponential as

$$
\frac{\mathrm{d} \bar{c}}{\mathrm{~d} t}=-\bar{c} \frac{Q}{V},
$$

where $V$ is the room volume. The mean concentration will decay with time as

$$
\frac{\bar{c}}{\bar{c}_{0}}=e^{-t / \tau_{w i n d}},
$$

where $\tau_{\text {wind }}=V / A^{*} U_{0}$ and $\bar{c}_{0}$ is the initial concentration within the room. This theory assumes that $\bar{c}=0$ in the environment (or that $\bar{c}$ refers to the concentration difference between the room and the ambient).

We can compare this model to the decay of the mean concentration in experiments for various imposed flow speeds $U_{0}$ (see figure $3 \mathrm{a}$, inset). When time is non-dimensionalised by $\tau_{\text {wind }}$ (using $C_{d}=0.6$ ), the data collapse and an exponential decay shows a good fit to the data (figure 3a). The slightly faster

decay at early times could be explained by the fact that the room is not perfectly mixed in the early stages of the experiment (i.e. the concentration is not uniform across the room). Vertical profiles of dye (Fig. 3b) showed a clear signature at early times $\left(t / \tau_{\text {wind }}=0.05\right)$ of the incoming (undyed) jet (also visible in figure $2 \mathrm{~b}$ ), while the profiles were observed to be close to well-mixed at later times. From this analysis we confirm that the pressure loss through the windows is well-described by $C_{d}=0.6$. This value of $C_{d}$ suggests that the thick wall of the model room does not have a significant effect on the flow, as $C_{d}=0.6$ is the value for a sharp-edged orifice.

\subsection{Exchange ventilation}

We now examine the opposite extreme, when ventilation is driven solely by an initial temperature difference between the interior of the room and the exterior. 

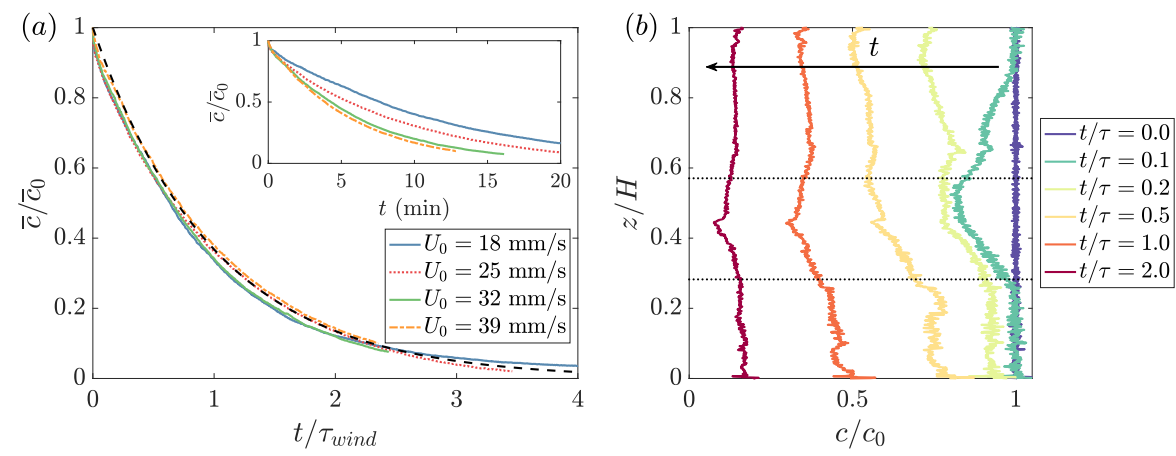

Figure 3: Wind-driven ventilation: (a) The decay of the mean concentration $\bar{c}$ of dye, with non-dimensional time $t / \tau_{\text {wind }}$, where $\tau_{\text {wind }}=V / A^{*} U_{0}$. The dashed line shows an exponential decay. The inset shows the decay of the mean concentration with dimensional time. (b) The horizontally averaged dye profile for $U_{0}=39 \mathrm{~mm} \mathrm{~s}^{-1}$.
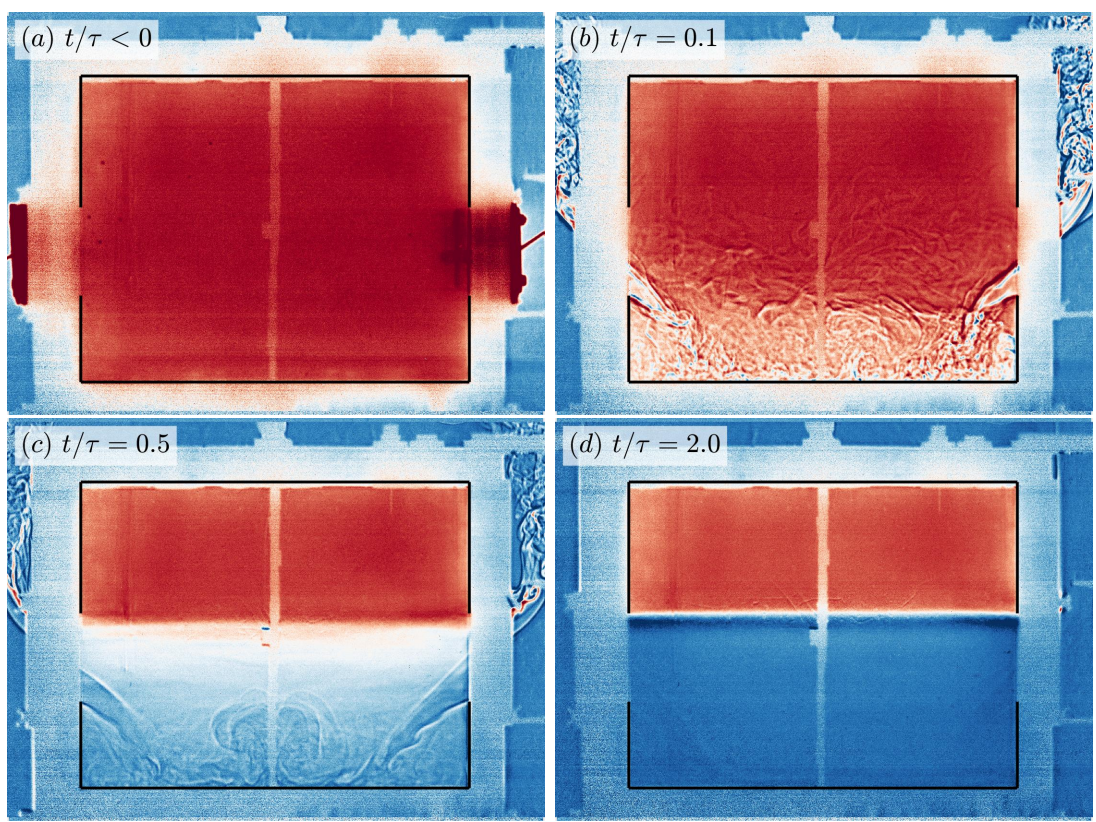

Figure 4: Buoyancy-driven ventilation. Images showing the model room (a) before the start of an experiment $\left(t / \tau_{e x}<0\right)$, (b) $t / \tau_{e x}=0.1$, (c) $t / \tau_{e x}=0.5$, (d) $t / \tau_{e x}=2.0$. The images are from experiment 8 from table A.1. False colour corresponds to intensity normalised by background intensity. Red corresponds to dyed warm fluid, while blue is undyed ambient fluid. The interior boundaries of the room and the windows are indicated with overlaid lines. 


\subsubsection{Well-mixed model}

There are two models that have been used to predict the ventilation flow due to a temperature difference between the inside and outside of a room. The first type of model is an orifice model, similar to that used in the previous section for wind-driven ventilation. For an orifice model, the flow rate is first determined by considering the pressure difference (as a function of height) between the inside and outside of the room and by using Bernoulli's theorem to calculate the velocity, neglecting any dissipation in the flow [5]. The dissipation is then accounted for by use of a discharge coefficient, $C_{d}$. The second type of model is known as a two-layer hydraulic model and predicts the flow by assuming it is hydraulically controlled at the opening [35]. A two-layer hydraulic model has the benefit that it does not assume that the flow is dissipationless and it correctly predicts that the exchange flow will not be symmetric for doorway flows, where it has been observed that the lower cool layer has a greater thickness than the upper warm layer. However, a hydraulic model does not account for all the dissipation in the flow, and an empirical constant is still often needed.

In this paper, we use an orifice model for exchange ventilation rather than a two-layer hydraulic model as this provides a more natural comparison between exchange ventilation and wind-driven ventilation, allowing us to interpolate smoothly between the two cases. We assume that the same discharge coefficient $\left(C_{d}=0.6\right)$ correctly accounts for the contraction of streamlines and turbulent dissipation in the case of exchange ventilation as was used in the previous section on wind-driven ventilation. This was found to be the case by Kiel, who found that $C_{d}=0.6$ for $1 \times 10^{4}<R e<15 \times 10^{4}$ in reduced-scale experiments similar to those performed here [30].

If the ventilation is solely driven by a temperature difference between the inside and outside of the room, the velocity through the window can be derived from Bernoulli's equation as $U(z)=\sqrt{2 g^{\prime} z}$, where $z$ is the distance from the neutral buoyancy height - the height at which the pressures inside and outside the room are equal - and $g^{\prime} \equiv g(\Delta \rho / \rho)$, is the reduced gravity across the window, where $\Delta \rho$ is the density difference due to temperature variations and $\rho$ is a reference density [5]. The density difference due to temperature variation is $\Delta \rho / \rho=\gamma \Delta T$, where $\Delta T$ is the temperature difference between the model room and the ambient and $\gamma$ is the thermal expansion coefficient $\left(\gamma=2.07 \times 10^{-2}\right.$ and $3.66 \times 10^{-3} \mathrm{~K}^{-1}$ for water and air, respectively). We will assume the neutral buoyancy height is at the centre of the window. Then the ventilation flow rate is given by

$$
Q=\frac{A^{*}}{h_{0}} \int_{0}^{h_{0} / 2} \sqrt{2 g^{\prime} z} \mathrm{~d} z=\frac{1}{3} A^{*} \sqrt{g^{\prime} h_{0}},
$$

where $h_{0}$ is the window height. 
In previous models, it has been assumed that the room is well mixed in the volume $V_{h}$ below the top of the windows [5]. With this well-mixed assumption, the reduced gravity next to the window will decay with time as

$$
\frac{\mathrm{d} g^{\prime}}{\mathrm{d} t}=-g^{\prime} \frac{n A^{*} \sqrt{g^{\prime} h_{0}}}{3 V_{h}},
$$

where $n$ is the number of windows, all of which are at the same height in the room. This leads to

$$
\frac{\Delta T}{\Delta T_{0}}=1-\frac{V_{h}}{V}+\frac{V_{h}}{V}\left(1+\frac{t}{\tau_{e x}}\right)^{-2}
$$

where $\Delta T_{0}$ is the initial mean temperature difference between the room and the exterior, and

$$
\tau_{e x}=\frac{6 V_{h}}{n A^{*} \sqrt{g_{0}^{\prime} h}} .
$$

We can compare this well-mixed model with experiments for various initial temperature differences (dash-dot line, figure 5a). We see that the well-mixed model gives a good prediction for the ventilation rates at early times, but underpredicts the ventilation rate at later times. By examination of the temperature profiles, we can see that the room is not well-mixed below the top of the window (figure $5 \mathrm{~b}$ ). This can also be seen in the images in figure 4 . In the following section, we will construct a new model that assumes there is no mixing between the flow through the window and the room. This will allow us to give an upper and lower bound for the evolution of the mean temperature within the room.

\subsubsection{Zero-mixing model}

If the room is not well mixed, the ventilation of the room below the top of the windows will take place in two stages. In the first stage, a constant flow rate

will fill up the volume of the room that sits below the bottom of the window, forming a two-layer stratification in the room. When the interface between the two layers reaches the bottom of the window, the system will transition to a second stage. In the second stage, the interface between the two layers moves up the height of the window. Below the interface, there is no density difference between the inside and outside of the window. We can therefore model this behaviour as if the window height were reducing with time. Once the interface rises to the top of the window, the exchange flow will cease and further reduction in the average temperature of the room will be due to diffusion of heat down from the upper layer. This final state can be observed in figure $4 \mathrm{~d}$.

Initially, the flow rate through the windows is constant and equal to

$$
Q=\frac{n}{3} A^{*} \sqrt{g_{0}^{\prime} h_{0}},
$$



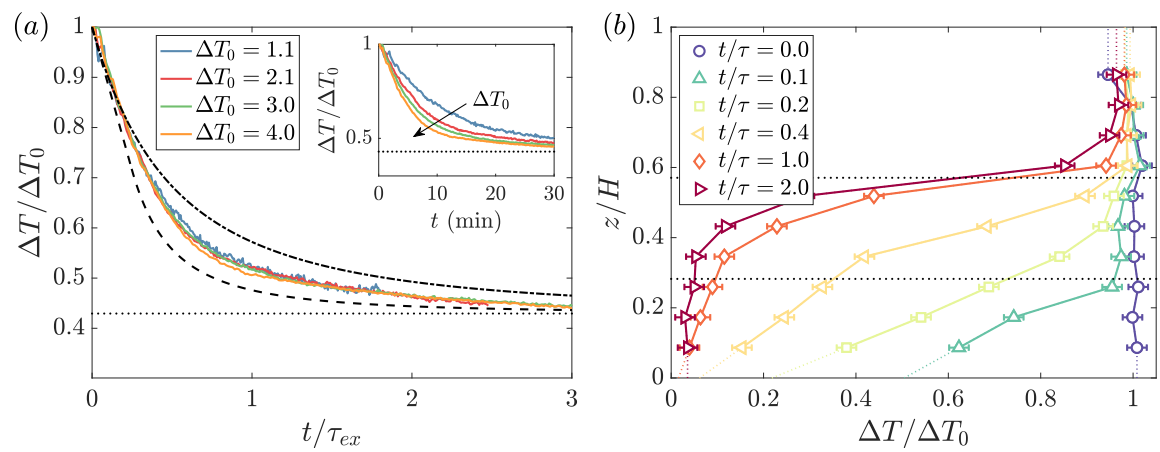

Figure 5: Buoyancy-driven ventilation. (a) The decay of the mean temperature in the model room with non-dimensional time $t / \tau_{e x}$, where $\tau_{e x}=3 V_{h} /\left(A^{*} \sqrt{g_{0}^{\prime} h}\right)$. The dash-dotted curve is the result of the well-mixed model (6) and the dashed curve is the zero mixing model (16). The horizontal dotted line at $\Delta T / \Delta T_{0}=0.43$ indicates the temperature in the room if $V_{h}$ was filled with ambient fluid. (b) The time evolution of temperature profiles within the room for $\Delta T_{0}=3 \mathrm{~K}$.

where $g_{0}^{\prime}$ is the initial reduced gravity. This leads to the mean temperature difference reducing linearly with time

$$
\frac{\Delta T}{\Delta T_{0}}=1-2 \frac{V_{h}}{V} \frac{t}{\tau_{e x}}, \quad \text { for } t<t_{L} .
$$

The volume $V_{l}$ of the room below the windows is filled with external fluid when

$$
t_{L}=\frac{V_{l}}{V_{h}} \frac{\tau_{e x}}{2} .
$$

When the interface reaches the bottom of the window, the effective window height $h$ reduces with time at a rate given by

$$
\frac{\mathrm{d} h}{\mathrm{~d} t}=-\frac{h_{0}}{V_{h}-V_{l}} Q .
$$

The flow rate through the window will vary with time as $Q$ is a function of $h$

$$
Q=\frac{n}{3} A^{*} \sqrt{g_{0}^{\prime} h_{0}}\left(\frac{h}{h_{0}}\right)^{3 / 2},
$$

where the effective window area is assumed to be equal to $A^{*} h / h_{0}$ (i.e. the window is rectangular). This leads to the differential equation

$$
\frac{\mathrm{d} h}{\mathrm{~d} t}=-\frac{h_{0}}{V^{*}} \frac{2}{\tau_{e x}}\left(\frac{h}{h_{0}}\right)^{3 / 2},
$$

where $V^{*}=\left(V_{h}-V_{l}\right) / V_{h}$. Using the boundary condition that $h=h_{0}$ at $t=t_{L}$ this gives

$$
\frac{h}{h_{0}}=\left(1+\frac{t-t_{L}}{V^{*} \tau_{e x}}\right)^{-2} .
$$


The rate of change of the mean reduced gravity is therefore

$$
\frac{\mathrm{d} g^{\prime}}{\mathrm{d} t}=-\frac{2 g_{0}^{\prime}}{\tau_{e x}} \frac{V_{h}}{V}\left(1+\frac{t-t_{L}}{V^{*} \tau_{e x}}\right)^{-3} .
$$

Using $g^{\prime} / g_{0}^{\prime}=1-V_{l} / V$ when $t=t_{l}$ and converting buoyancy into temperature, we find

$$
\frac{\Delta T}{\Delta T_{0}}=1-\frac{V_{h}}{V}+\frac{V_{h}-V_{l}}{V}\left(1+\frac{t-t_{L}}{V^{*} \tau_{e x}}\right)^{-2}, \quad \text { for } t>t_{L} .
$$

We can compare this model to the experimental results (dashed line, figure 5a). We see that a zero-mixing model more closely predicts the behaviour of the temperature decay in the room than the well-mixed model, in that the experiments show evidence of an initial linear decay of the temperature in the room, which is described by the zero-mixing model. The results lie between the well-mixed and zero-mixing model, which would be expected from the temperature profiles in figure $5 \mathrm{~b}$, which show that the temperature gradient in the lower layer is approximately linear, indicating that some mixing has occurred.

The model developed in this section is similar to that developed by Phillips and Woods that assumed no mixing between a buoyancy-driven exchange flow entering a room through a doorway [6]. However, their model assumed that the ventilation rate did not change as the interface moved up over the opening, with the neutral buoyancy height in the opening remaining constant, until the height of the interface within the room reached the neutral buoyancy height in the doorway. In contrast, the effective height of the window in our model decreases continuously as the interface moves over the opening height.

\subsection{Combined ventilation}

When both temperature and thermal effects are significant, we can characterise the relative importance of these effects on the flow through the windows by an initial Froude number given by

$$
F r_{0}=\frac{U_{0}}{\sqrt{g_{0}^{\prime} h_{0}}}=\frac{U_{0}}{\sqrt{g h_{0} \gamma \Delta T_{0}}},
$$

where $U_{0}$ is the (uniform) wind speed, $g_{0}^{\prime}$ is the initial reduced gravity of the room relative to the environment and $h_{0}$ is the height of the window. As we shall see, the Froude number is a appropriate non-dimensional parameter to describe the switch from buoyancy-dominated to wind-dominated behaviour. When $F r_{0}>1$, we expect the flow through the windows to be wind-dominated, whereas if $F r_{0}<1$, we expect (at least initially) the flow to be dominated

by buoyancy. Ventilation of the room will decrease the temperature difference 
between the room and the ambient, reducing $g^{\prime}$. This means that although the Froude number may start small, unless $U_{0}=0$ warm fluid will be flushed from the room by the ventilation, the Froude number will increase and the ventilation eventually becomes wind-dominated.

The Froude number is the ratio of the velocity associated with the wind $U_{0}$, to the velocity associated with the buoyancy-driven flow, $\sqrt{g_{0}^{\prime} h_{0}}$. The Froude number is related to the Archimedes number $A r=1 / F r^{2}$, a parameter that has also been used to describe the interaction of wind and buoyancy effects,

$$
A r=\frac{\Delta \rho g h_{0}}{\rho U_{0}^{2}}
$$

which is proportional to the ratio of the hydrostatic pressure, $\Delta \rho g h_{0}$, to the dynamic pressure $\frac{1}{2} \rho U_{0}^{2}$.

\subsubsection{Wind dominated: $F r_{0}>1$}

When the initial Froude number $F r_{0} \gg 1$, the wind-driven flow will dominate the buoyancy-driven flow and we expect to get only in-flow through the

windward window and only out-flow through the leeward window. Images of the ventilation flow and the evolution of the interior stratification for $F r_{0}=1.22$ can be seen in figure 6 , which confirm this uni-directional flow. However, we see that, even for relatively small temperature differences, the behaviour of winddominated ventilation is quite different from wind-only ventilation (figure 2). The ventilation appears to occur in two stages: first $(t / \tau \lesssim 1)$ the lower part of the room is ventilated, up to the top of the windows (figure $6 \mathrm{~d}$ ), then $(t / \tau \gtrsim 1$ ) the upper part of the room is removed over a much longer timescale (figure $6 \mathrm{f}$ ).

We start by building a model for the ventilation rate. If we assume that the neutral buoyancy height remains at the centre of the window, Bernoulli's equation tells us that the velocity profile in the window is

$$
U(z)=\sqrt{U_{0}^{2}-2 g^{\prime} z}
$$

noting that because $F r_{0}>1 \Longrightarrow U_{0}^{2}-2 g^{\prime} z>0$ for $-h_{0} / 2<z<h_{0} / 2$. Integrating across the window, we find the flow rate through the window is

$$
Q=\frac{1}{3} A^{*} \sqrt{g^{\prime} h_{0}}\left(\left(F r^{2}+1\right)^{3 / 2}-\left(F r^{2}-1\right)^{3 / 2}\right)
$$

where $F r=U_{0} / \sqrt{g^{\prime} h_{0}}$ is the instantaneous Froude number (since $g^{\prime}$ decreases with time). We can compare this to the flow rate $Q_{\text {wind }}=A^{*} U_{0}$ for wind-driven ventilation (infinite $F r$ )

$$
\frac{Q}{Q_{\text {wind }}}=\frac{1}{3} \frac{\left(\left(F r^{2}+1\right)^{3 / 2}-\left(F r^{2}-1\right)^{3 / 2}\right)}{F r} .
$$



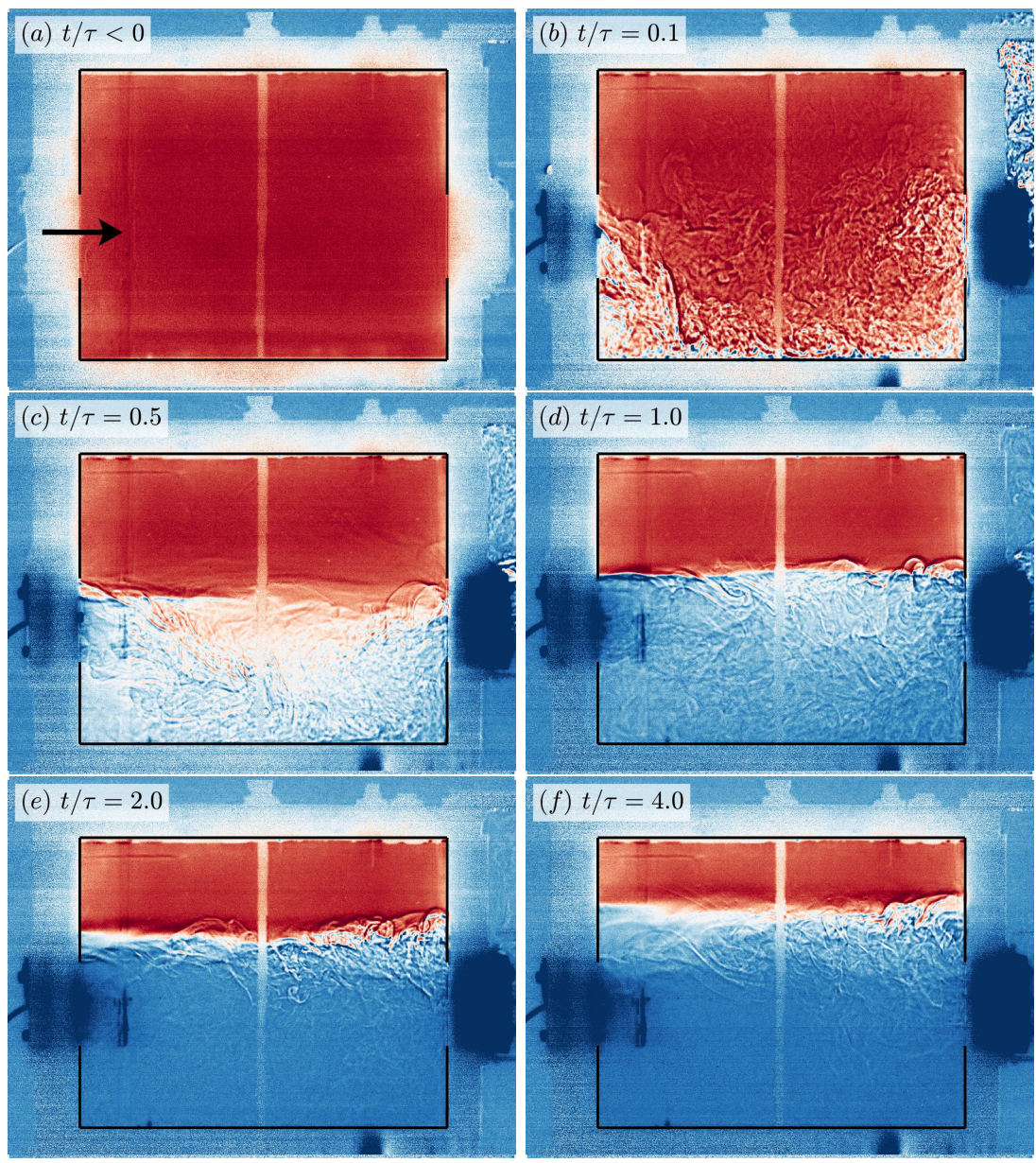

Figure 6: Wind-dominated combined ventilation $\left(F r_{0}=1.22\right)$. The images are from experiment 11 from table A.1. False colour corresponds to intensity normalised by background intensity. Red corresponds to dyed warm fluid, while blue is undyed cooler ambient fluid. The external flow is from left to right, as indicated by the arrow in $(a)$. The interior boundaries of the room and the windows are indicated with overlaid lines. 

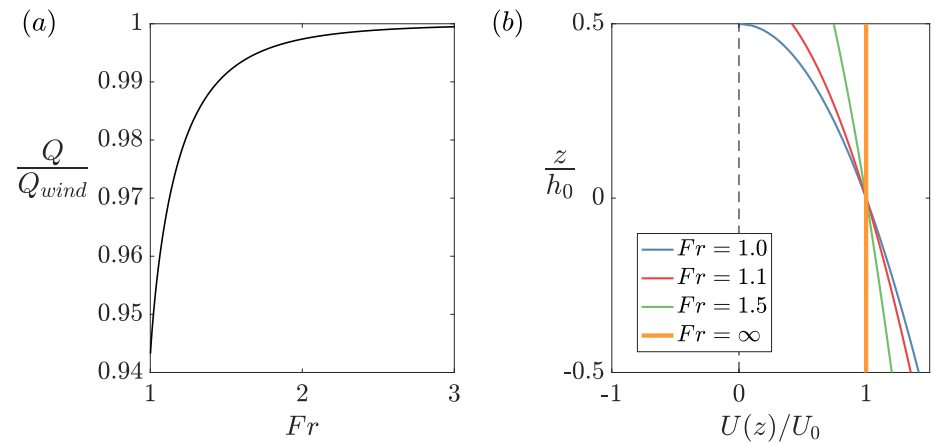

Figure 7: Temperature differences have little effect on wind-driven cross-ventilation. $(a)$ Ventilation flow rate as a function of $F r$ for $F r>1$, relative to ventilation flow rate for wind-only ventilation. For a room initially at a higher temperature than the external air but supplied with no additional heating the system will follow the trajectory from low to high $\mathrm{Fr}$. (b) Velocity profile for the windward-window for varying $F r$.

This normalised flow rate is plotted in figure 7. It can be seen that for $\mathrm{Fr}>1$, the effect of temperature is small. Even at $F r=1$ when the wind-driven and stack-driven ventilation forces balance, the ventilation flow rate is $2 \sqrt{2} / 3 \approx 94 \%$ of wind-only ventilation. Therefore, modelling the ventilation flow rate through the window as being due to wind only will lead to at most a $6 \%$ error. This error will also decrease with time as the ventilation of the room will reduce $g^{\prime}$ and increase $\mathrm{Fr}$.

Given that the effect of temperature on the flow rate is so small, it is worth comparing the wind-dominated experiments with a simple exponential model that assumes the room is well-mixed in the reduced volume $V_{h}$. The decay of temperature in the model room for experiments at various $F r_{0}>1$ is plotted in figure 8a. Time is non-dimensionalised by $\tau_{\text {wind }}=V /\left(A^{*} U_{0}\right)$, which gives a good collapse for the data. Two models are plotted for comparison: an exponential decay that assumes the room is well-mixed over the entire room volume $V$ (dashed line) or well-mixed only below the top of the windows $V_{h}$ (dashed-dot line). The effective volume of the room appears to be close to $V_{h}$ and does not vary significantly across this range of Froude numbers.

Although the initial decay of the temperature difference in the room approximately follows the exponential fit with a timescale set by $V_{h}$, the experiments appear to have a slightly slower ventilation rate than the model would suggest. Inspection of figures $6 \mathrm{~b}$ and $\mathrm{c}$ (and the supplementary video) shows that the inflow through the window initially does not mix well with the room volume and the momentum of the inflow allows it to cross the floor of the room, exiting via the leeward window. This short-circuiting means that the fluid exiting the 

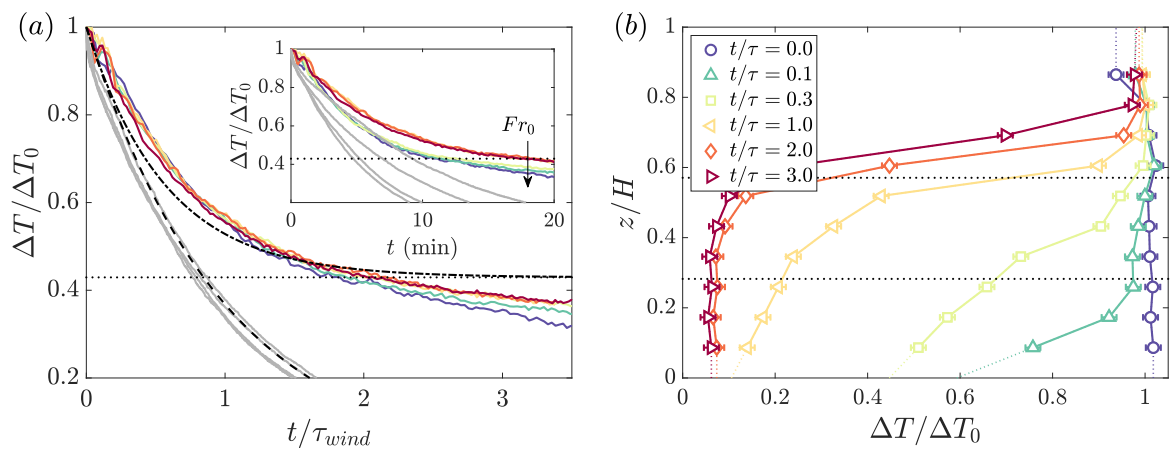

Figure 8: Experimental results for wind-dominated ventilation $\left(F r_{0}>1\right)$. (a) The decay of the mean temperature in the model room with non-dimensional time $t / \tau_{\text {wind }}$, where $\tau_{\text {wind }}=$ $V /\left(A^{*} U_{0}\right)$. Two models are plotted: an exponential decay, assuming the room is well-mixed below the top of the windows $V_{h}$ (dash-dot line) or well-mixed over the room volume $V$ (dashed line). Wind-only experiments (as previously plotted in figure 3a) are plotted in grey for comparison. The dotted line indicates the temperature difference in the room if $V_{h}$ were filled with ambient fluid. (b) The evolution of the temperature profiles within the room for $F r=1.0$. The dotted lines indicate the top and bottom of the window.

room through the leeward window is at a lower temperature, as compared to the temperature of fluid that would leave the room if $V_{h}$ were well-mixed. This reduces the rate at which the temperature decreases.

\subsubsection{Thermally dominated: $F r_{0}<1$}

Images of the ventilation flow for $F r_{0}=0.61$ are shown in figure 9. For $F r_{0}<1$ there is the potential for both in-flow and out-flow through both openings, and this two-way exchange flow through each window is observed in this experiment. Consequently, there will be a vertical location $\hat{z}$ on each window, where the wind and buoyancy cancel each other and $U(\hat{z})=0$. The net flow-rate out of the room will be the total out-flow through all windows.

For the window on the windward side, wind will oppose the buoyancy-driven out-flow through the top of the window. Using Bernoulli's equation, the vertical location where $U=0$ is $\hat{z}=U_{0}^{2} / 2 g^{\prime}$, and out-flow through the windward window will occur for $z>\hat{z}$, for which $U(z)=\sqrt{2 g^{\prime} z-U_{0}^{2}}$.

For the window on the lee side, wind will reinforce the out-flow through the top of the window. The vertical location where $U=0$ is $\hat{z}=-U_{0}^{2} / 2 g^{\prime}$, and out-flow through the window will occur for $z>\hat{z}$, for which $U(z)=\sqrt{2 g^{\prime} z+U_{0}^{2}}$.

The total ventilation flow rate is, therefore,

$$
\begin{gathered}
Q=\frac{A^{*}}{h} \int_{U_{0}^{2} / 2 g^{\prime}}^{h / 2} \sqrt{2 g^{\prime} z-U_{0}^{2}} \mathrm{~d} z+\frac{A^{*}}{h} \int_{-U_{0}^{2} / 2 g^{\prime}}^{h / 2} \sqrt{2 g^{\prime} z+U_{0}^{2}} \mathrm{~d} z \\
=\frac{1}{3} A^{*} \sqrt{g^{\prime} h_{0}}\left(\left(1-F r^{2}\right)^{3 / 2}+\left(1+F r^{2}\right)^{3 / 2}\right) .
\end{gathered}
$$



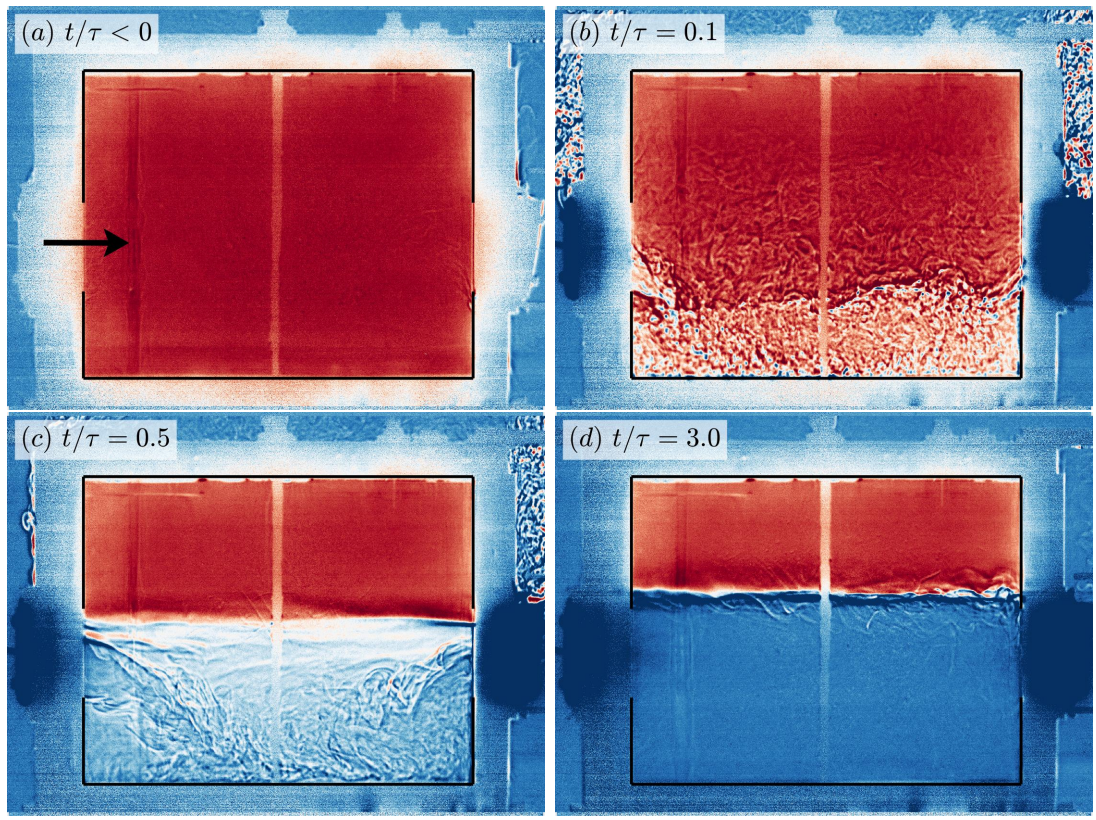

Figure 9: Buoyancy-dominated combined ventilation $\left(F r_{0}=0.61\right)$ : The images are from experiment 18 from table A.1. False colour corresponds to intensity normalised by background intensity. Red corresponds to dyed warm fluid, while blue is undyed cooler ambient fluid. The external (wind) flow is from left to right, as indicated by the arrow in $(a)$. The interior boundaries of the room and the windows are indicated with overlaid lines. 

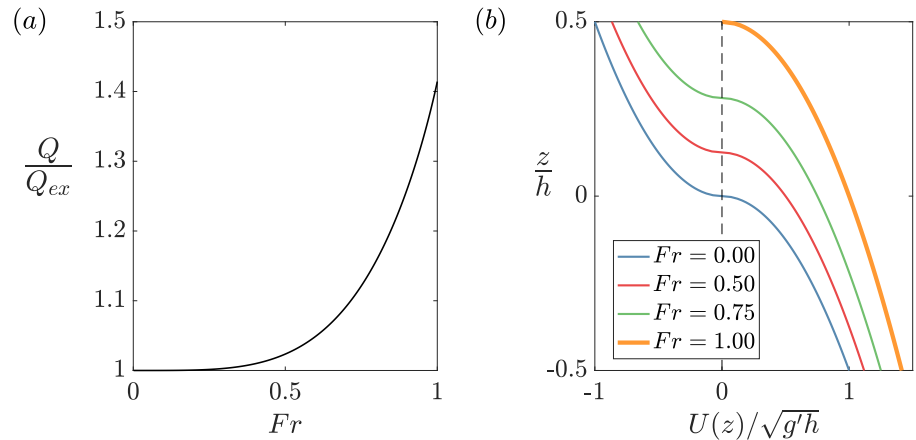

Figure 10: Wind enhances buoyancy-driven exchange ventilation. (a) Ventilation flow rate as a function of $F r$ for $0<F r<1$, relative to ventilation flow rate for buoyancy-driven exchange flow. (b) Velocity profiles at the windward-window for various $\mathrm{Fr}$.

We can compare this ventilation flow rate to that which occurs for buoyancydriven exchange flow through two windows, $Q_{e x}$. This is the flow rate $Q$ given by equation (4) (multiplied by two as there are two windows),

$$
Q_{e x}=\frac{2}{3} A^{*} \sqrt{g^{\prime} h_{0}}
$$

The normalised ventilation rate is therefore

$$
\frac{Q}{Q_{e x}}=\frac{1}{2}\left(\left(1-F r^{2}\right)^{3 / 2}+\left(1+F r^{2}\right)^{3 / 2}\right) .
$$

This normalised ventilation rate is plotted in figure 10. As expected, for $F r=0$, which corresponds to exchange ventilation, $Q / Q_{e x}=1$. As $F r$ approaches 1 , the ventilation flow rate increases by a factor of $\sqrt{2} \approx 1.4$.

The evolution of the mean temperature in the model room for various initial Froude numbers is plotted in figure 11a (inset). If time is non-dimensionalised by the time-scale associated with exchange flow and normalised by the initial ratio of the ventilation flow rates, (25) with $F r=F r_{0}$, the data collapse. The zero-mixing and complete mixing exchange flow models provide good bounds on the temperature within the room for the initial decay.

Strictly speaking, by normalising by the initial ratio of the ventilation flow rates, we have only made the initial gradients of the temperature decay collapse. The instantaneous decay of $\Delta T / \Delta T_{0}$ is determined by the instantaneous Froude number at the window, which will reduce with time as the ventilation progresses. As these experiments begin with different Froude numbers and the ventilation has a nonlinear dependence on Froude number, the instantaneous Froude number, $\operatorname{Fr}(t)$, will decay at a different rate for experiments with different $F r_{0}$. We have not included integrated models that take account of $F r(t)$, as these will also only provide bounds on the time evolution of the system. 

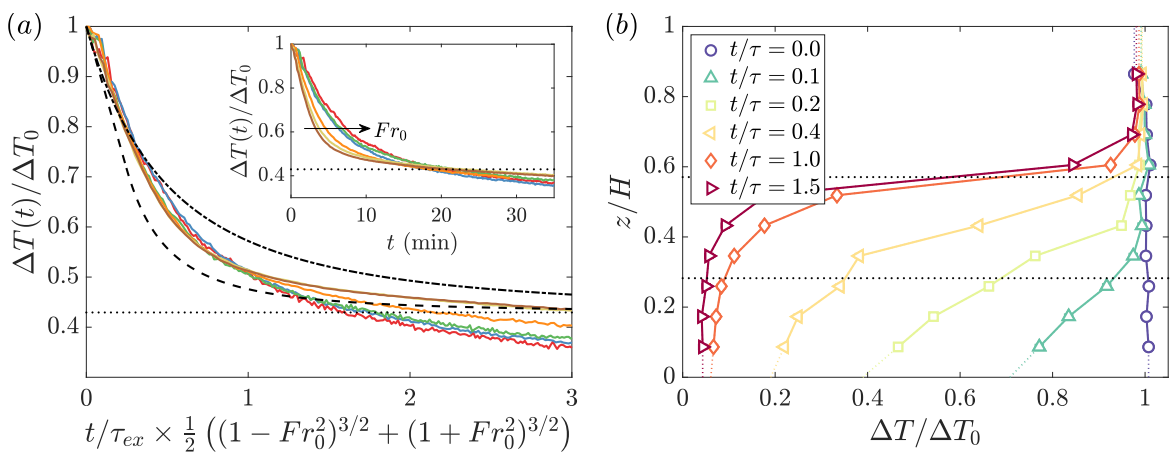

Figure 11: Buoyancy-dominated ventilation $\left(F r_{0}<1\right)$. (a) The decay of the mean temperature in the model room with non-dimensional time $t / \tau_{e x}$, where $\tau_{e x}=3 V_{h} / A^{*} \sqrt{g_{0}^{\prime} h}$. The thin dotted line indicates the temperature in the room if $V_{h}$ were filled with ambient fluid. (b) The evolution of the temperature profiles within the room for $F r_{0}=0.61$.

\section{Late-time behaviour}

For all of the combined wind and buoyancy experiments performed for this study, once the layer of fluid below the top of the windows is replaced by fluid of the same temperature as that outside the room, the system transitions to what we will call the late-time behaviour. Flow through the windows is entirely wind driven and mixing is produced by the jet that comes in through the window and induces shear in the interface between the two layers. Shear-driven mixing is by no means a solved problem. However, we can develop some simple scaling arguments for the present case. We will assume that the density gradient in the mixing layer $\nabla \rho \sim \Delta \rho / \delta$, where $\delta$ is the thickness of the mixing layer, and $\Delta \rho=\gamma \Delta T_{\delta}$ is the density difference across the mixed layer. We will assume that the shear scales as $\nabla u \sim U_{0} / \delta$. We can use these scalings to construct a local Richardson number

$$
R i=\frac{g \nabla \rho}{\rho(\nabla u)^{2}}=\frac{g^{\prime} \delta}{U_{0}^{2}}=\frac{1}{F r_{\delta}^{2}},
$$

where $F r_{\delta}$ is the $F r$ based on the length scale $\delta$.

We define the dimensionless entrainment rate $E=Q_{e} / U_{0} A_{f}$, where $Q_{e}$ is the volume flux across the interface and $A_{f}$ is the floor area of the room. We can re-write the entrainment as

$$
E=\frac{Q_{e}}{U_{0} A_{f}}=-\frac{1}{U_{0}} \frac{\mathrm{d} D}{\mathrm{~d} t}
$$

where $D$ is the depth of the upper layer, and we have assumed that entrainment is occurring over some constant fraction of the interface. 
It is usual to relate entrainment to the Richardson number in a power law of the form

$$
E \sim R i^{m},
$$

where $m$ is some power. Equivalently, we could write $E \sim F r_{\delta}^{n}$, where $n=-2 m$. Schneider [36] investigated the mixing produced by a jet parallel to a density interface and found $n=3$. This is the same power law as that found by Baines, who studied the mixing induced by a jet impinging on (perpendicular to) a density interface [37]. Alternatively, Herault et al. found $E \sim F r^{3}$ for $F r \ll 1$ and $E \sim F r$ for $F r \gg 1$ for a jet impinging on a density stratification [38]. A formulation that takes account of confinement has also been suggested, with $E \sim F r^{2}$ for the unconfined case and $E \sim F r^{3}$ for the confined case, with the difference due to the influence of secondary flows for the confined case [39].

Before measuring the entrainment, we will examine the interface thickness $\delta$. To measure the interface thickness, we fitted a hyperbolic tangent profile to the temperature profile in the model room. This was done for $t=t_{0}$, the time at which $\Delta T / \Delta T_{0}=1-V_{h} / V$ (i.e. the temperature of the room if the volume below the windows were replaced by ambient fluid). An example of the fit of a tanh profile is shown in figure 12a. The variation of $\delta$ at $t_{0}$ with initial Froude number $F r_{0}$ is plotted in figure $12 \mathrm{~b}$, which shows that as $F r_{0}$ increases, and wind dominates over buoyancy, the interface thickness increases. Error bars are calculated from $95 \%$ confidence intervals of the coefficient from the tanh fit. This measurement of interface thickness is thicker than would be inferred from shadowgraph images. This increased thickness is likely due to a combination of the effect of waves on the interface and the time constant of the thermistor (3 seconds). Unfortunately, it was not possible to make an accurate measurement of the interface thickness from shadowgraph images due to the distortion created by the strong refractive index variation at the interface, and the fact that the image represents an integral of the deviations of the light across the full width of the tank. Consequently, we use the measurements of $\delta$ from the fitted profiles to calculate $F r_{\delta}$ and examine entrainment.

The late-time behaviours of the experimental results are plotted in figure 13a, which shows $\Delta T / \Delta T_{0}$ shifted by $t_{0}$. We could use these data to find the entrainment rate provided only the entrainment was responsible for reducing the mean temperature. However, for the lower Froude number experiments, heat loss from the room accounts for up to $1 / 3$ of the rate of temperature decay (calculated by examining the rate of decrease in temperature of the top thermistor). Instead, we use the midpoint of the tanh fit to find the depth of the upper layer $D$. The depth of the upper layer $D$ relative to $D_{0}$, the depth at $t_{0}$, is plotted in figure $13 \mathrm{~b}$. We find $\mathrm{d} D / \mathrm{d} t$ by fitting a line to $t>t_{0}$ and 

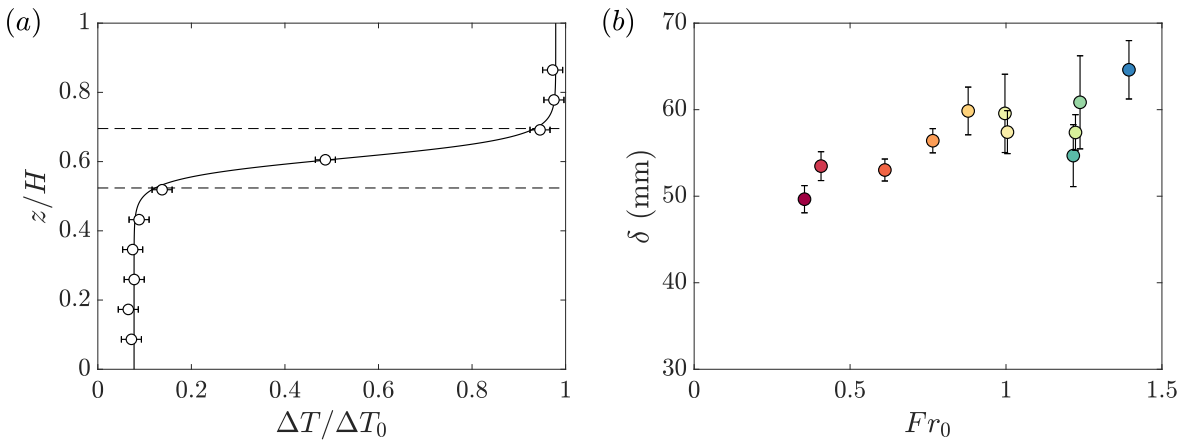

Figure 12: Interface thickness. (a) A tanh profile (solid line) is fitted to the temperature measurements (circles) at $t=t_{0}$, the time at which $\Delta T / \Delta T_{0}=1-V_{h} / V$. This profile is used to estimate the interface thickness $\delta$ (indicated with dashed lines). The example shown is for $F r=1.0$. (b) The interface thickness $\delta$ at $t_{0}$ with initial Froude number $F r_{0}$. Error bars are calculated from $95 \%$ confidence intervals of the coefficient from the tanh fit.
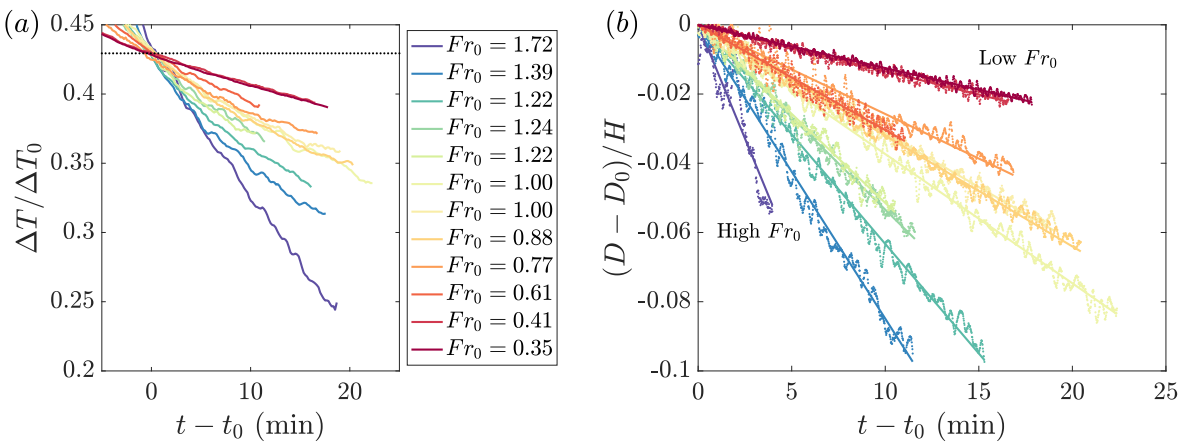

Figure 13: Late time behaviour. (a) All wind-buoyancy combined experiments, shifted by $t_{0}$, the time at which $\Delta T / \Delta T_{0}=1-V_{h} / V$. (b) The depth of the upper layer $D$ found by fitting a tanh function to the temperature profile (points) and a linear fit (lines).

calculate the entrainment $E$ from (27). The entrainment $E$ is plotted against $F r_{\delta}$ in figure 14. The density difference used to calculate $F r_{\delta}$ is the density difference across $\delta$, measured from the same tanh fit. The data suggest a linear relationship between entrainment and $F r_{\delta}$, although a greater range of $F r_{\delta}$ would be needed for a definitive answer.

From these results we can see that there are two processes that occur during the ventilation of the model room. For the experiments described above, the layer below the top of the windows is removed initially and then the remaining upper layer is eroded. At low Froude numbers, the erosion of the upper layer occurs much more slowly than the removal of the lower layer. As the Froude number increases, however, this balance could shift. The timescale associated 


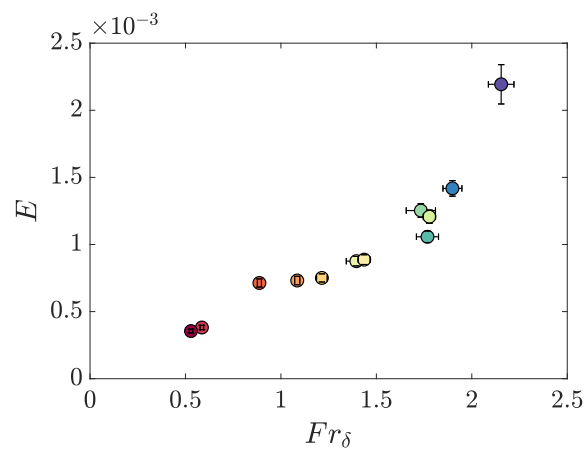

Figure 14: The entrainment $E=\left(1 / U_{0}\right) \times \mathrm{d} D / \mathrm{d} t$, where $\mathrm{d} D / \mathrm{d} t$ is found from a linear fit to the data plotted in 13(b). Vertical error bars are two standard deviations of the coefficient of the linear fit of $D$. Horizontal error bars are calculated from the $95 \%$ confidence intervals of $\delta$.

with removal of the lower layer is

$$
\tau_{\text {wind }}=\frac{V_{h}}{A^{*} U_{0}},
$$

whereas the timescale associated with the removal of the upper layer is

$$
\tau_{\text {entrain }}=\frac{D}{U_{0} E},
$$

where we have assumed that $E$ is a constant (i.e. it does not vary with $D$ ). This appears reasonable for the experiments in figure $13 a$ (as they have constant gradients), but might vary when $D$ becomes small or the aspect ratio of the room increases.

We are interested in determining the critical Froude number $F r_{c}$, for which $\tau_{\text {wind }}=\tau_{\text {entrain }}$, as then for $F r \ll F r_{c}$, ventilation will occur in two stages and temperature differences would need to be taken into account, whereas for $F r \gg F r_{c}$, the cross-ventilation can be treated as purely wind-driven. This means we seek the Froude number that gives the critical entrainment rate of

$$
E_{c}=\frac{D A^{*}}{V_{h}},
$$

which depends only on the room geometry. If we assume that the entrainment rate scales linearly with Froude number, fitting a function of the form $E=a F r_{\delta}$ gives for the constant coefficient $a=7.4 \times 10^{-4}$. Using the geometry of our model room gives $E_{c}=0.02$, which allows us to estimate the critical $F r_{\delta}=28$. Figure $12 b$ suggests that $\delta$ is a weak function of $F r_{0}$, so we estimate $F r_{0} \approx b F r_{\delta}$, finding the coefficient $b=0.72$ from a fit to the data. An estimate for the 
critical initial Froude number for this room geometry is then $F r_{0} \approx 20$. For $F r_{0} \ll 20$, we expect to see a two-stage ventilation of the room, whereas for $F r_{0} \gg 20$ we expect that temperature is not significant and cross-ventilation can be modelled as driven by wind alone. This critical Froude number is specific to this room geometry. This analysis is meant to be suggestive, rather than to be taken literally, as it was necessary to extrapolate from the data available to calculate the estimate for the critical $F r_{c}$.

It should be noted that in formulating (30) we assumed that the velocity difference across the shear layer was equal to $U_{0}$, which will only be true when $F r_{0}$ is high or at late times for wind-driven ventilation. At lower $F r_{0}=O(1)$, the velocity at the top of the window will be suppressed at early times, reducing the entrainment rate from the upper layer. We should also be careful when using (31) if $D$ is close to zero, as the layer might overturn, changing the mixing dynamics. Experiments at intermediate Froude numbers are needed to progress further. As these experiments would require a new experimental apparatus, we leave them for future research.

\section{Conclusions}

Using experiments and simple theory, we have identified the effect of an initial temperature difference on the transient ventilation of an unobstructed crossventilated room with identical openings in the windward and leeward façades. The relative strengths of the wind-driven and buoyancy-driven flows are characterised by a Froude number $F r$, which is the ratio of the wind speed to a buoyancy-driven flow speed. For initially wind-dominated ventilation $\left(F r_{0}>1\right)$, a temperature difference makes only a minor modification to the inflow through the window and results in only a small decrease $(<6 \%)$ in the ventilation flow rate through the windows. This effect can be neglected in most practical applications. On the other hand, for initially buoyancy-dominated ventilation $\left(0<F r_{0}<1\right)$, the wind significantly changes the inflow through the window and, therefore, increases the ventilation flow rate by up to $40 \%$.

For the combined (wind and buoyancy) experiments described here, the ventilation occurs in two stages. In the first stage, the layer below the top of the windows is removed relatively rapidly. In the case of wind-dominated ventilation, this can be described by an exponential decay with a reduced room volume corresponding to the volume of the room below the top of the windows. For buoyancy-dominated ventilation, the decay of the mean temperature in a room can be modelled as exchange ventilation with a correction factor that is a function of the initial Froude number. 
The second stage of ventilation is the erosion of an upper layer (above the top of the windows) by entrainment into the wind-driven jet that crosses the room. The entrainment appears to follow a linear behaviour with a Froude number defined using the thickness of the mixing layer, the wind velocity and the buoyancy of the upper layer.

This research has two consequences for natural ventilation design. Firstly, in practice it appears that wind can have a significant effect on buoyancy-driven exchange flows through openings when cross-ventilation can occur, but buoyancy effects can be neglected when calculating flow rates when ventilation is dominated by wind. Secondly, even when buoyancy effects can be neglected when calculating the ventilation rate through an opening, temperature effects can still result in a stable stratification that can remain for a significant length of time (as compared to the time taken to remove fluid from below the top of the windows). If the main form of ventilation is by cross-ventilation, this could result in the build-up of contaminants above the top of the windows.

We have examined the case of cross-ventilation through two large openings, that are without obstructions, such as the lower half of a sash window that is fully opened. Different opening types would be expected to have an effect, particularly if the window has a vertical or horizontal hinge. This effect might be characterised by use of a new discharge coefficient.

Several open questions remain. One of the most significant relates to the transition between the two stages of ventilation. We have proposed some simple scaling arguments to identify the critical Froude number at which temperature effects can be neglected and the ventilation modelled as entirely wind-driven, but further experiments at intermediate Froude numbers are needed, as are experiments that vary the room geometry. Further investigation of the entrainment at late times are also of interest, to identify whether the entrainment rate changes as the interface moves away from the top of the windows and whether the entrainment continues to follow a linear relationship with Froude number at higher Froude numbers.

In this study, we have examined the transient case of a room that cools down over time. An interesting area for future research would be the steady state of a room with cross-ventilation and a heated floor (perhaps due to solar radiative heating of the floor). The effect of a combination of cross-ventilation and discrete heat gains in a larger room is another important area for future research, as discrete heat gains could result in horizontal temperature variations.

A final significant effect that we have not examined here is the effect of turbulence. Turbulence would be expected to disrupt the exchange flow, decreasing the effective discharge coefficient due to mixing between the incoming and outgoing flows. Further experiments at higher Reynolds numbers and at full scale 
would also be of interest.

\section{Acknowledgements}

This work is supported by the Engineering and Physical Sciences Research Council (EPSRC) Grand Challenge grant Managing Air for Green Inner Cities (MAGIC) [grant number EP/N010221/1]. We are very grateful to the technical staff in the G. K. Batchelor Laboratory, and particularly David Page-Croft, for their expert work in designing and commissioning the flume and for the construction of the other experimental apparatus. We thank Dr Jamie Partridge for his help with the temperature measurements. We also thank Nouhaila Fahdi for assisting with experiments.

\section{Declaration}

All authors have approved the final version of the manuscript being submitted. This article is the authors' original work, has not received prior publication and is not under consideration for publication elsewhere. There are no known conflicts of interest. 


\section{Appendix A. Experiments}

\begin{tabular}{cccc}
\hline Experiment & $U_{0}(\mathrm{~mm} / \mathrm{s})$ & $\Delta T(\mathrm{~K})$ & $F r_{0}$ \\
\hline 1 & 18 & 0 & $\infty$ \\
2 & 25 & 0 & $\infty$ \\
3 & 32 & 0 & $\infty$ \\
4 & 39 & 0 & $\infty$ \\
\hline 5 & 0 & 1.1 & 0 \\
6 & 0 & 2.1 & 0 \\
7 & 0 & 3.0 & 0 \\
8 & 0 & 4.0 & 0 \\
\hline 9 & 35 & 2.0 & 1.72 \\
10 & 35 & 3.0 & 1.39 \\
11 & 35 & 4.0 & 1.22 \\
12 & 25 & 2.0 & 1.24 \\
13 & 25 & 2.0 & 1.22 \\
14 & 25 & 3.0 & 1.00 \\
15 & 20 & 2.0 & 1.00 \\
16 & 25 & 3.9 & 0.88 \\
17 & 20 & 3.5 & 0.77 \\
18 & 25 & 8.0 & 0.61 \\
19 & 20 & 12.3 & 0.41 \\
20 & 20 & 16.3 & 0.35 \\
\hline
\end{tabular}

Table A.1: List of experiments 


\section{Appendix B. Heat loss through walls}

The rate of heat loss through the walls of the model room can be modelled using Newton's law of cooling, which states that

$$
\dot{T}_{\text {cool }}=\frac{\mathrm{d} \Delta T}{\mathrm{~d} t}=-\frac{\Delta T}{\tau_{\text {cool }}},
$$

where $\tau_{\text {cool }}=\rho V c_{p} / S U, S$ is the internal surface area of the box, $U$ is the heat transfer coefficient, and $c_{p}=4181 \mathrm{~J} / \mathrm{kg} \mathrm{K}$ is the heat capacity [40]. From experiments conducted by Partridge et al, we know that $U=5.5 \mathrm{~W} \mathrm{~m}^{-2}$ for the model room used [26]. Using this value of $U$ and the internal surface area of the box, we find $\tau_{\text {cool }}=5 \times 10^{4} \mathrm{~s}$.

We can construct a similar equation for the buoyancy-driven ventilation case by rearranging equation (5)

$$
\dot{T}_{e x}=\frac{\mathrm{d} \Delta T}{\mathrm{~d} t}=-\frac{2}{\tau_{e x}} \frac{(\Delta T)^{3 / 2}}{\left(\Delta T_{0}\right)^{1 / 2}} .
$$

The ratio of these two rates is

$$
\frac{\dot{T}_{\text {cool }}}{\dot{T}_{\text {ex }}}=\frac{\tau_{e x}}{2 \tau_{\text {cool }}}\left(\frac{\Delta T_{0}}{\Delta T}\right)^{1 / 2} .
$$

As $\tau_{e x}$ is inversely proportional to the square-root of the initial temperature difference, we can re-write this as

$$
\frac{\dot{T}_{\text {cool }}}{\dot{T}_{e x}}=\frac{C}{(\Delta T)^{1 / 2}}
$$

where the constant $C=0.0154 \mathrm{~K}^{1 / 2}$ for the setup in the current experiment. The heat lost through the walls will be less than $10 \%$ of the heat lost by buoyancydriven ventilation so long as the temperature difference $\Delta T>0.02 \mathrm{~K}$.

\section{References}

[1] L. Prez-Lombard, J. Ortiz, C. Pout, A review on buildings energy consumption information, Energy and Buildings 40 (3) (2008) 394-398. doi: $10.1016 / \mathrm{j}$. enbuild.2007.03.007.

[2] M. Ohba, I. Lun, Overview of natural cross-ventilation studies and the latest simulation design tools used in building ventilation-related research, Advances in Building Energy Research 4 (1) (2010) 127-166. doi:10.3763/ aber.2009.0405. 
[3] D. Etheridge, A perspective on fifty years of natural ventilation research, Building and Environment 91 (2015) 51-60. doi:10.1016/j.buildenv. 2015.02 .033 .

[4] F. Allard, Y. Utsumi, Airflow through large openings, Energy and Buildings 18 (2) (1992) 133-145. doi:10.1016/0378-7788(92) 90042-F.

[5] W. G. Brown, K. R. Solvason, Natural convection through rectangular openings in partitions1: Vertical partitions, International Journal of Heat and Mass Transfer 5 (9) (1962) 859-868. doi:10.1016/0017-9310(62) 90184-9.

[6] J. C. Phillips, A. W. Woods, On ventilation of a heated room through a single doorway, Building and Environment 39 (3) (2004) 241-253. doi: 10.1016/j. buildenv.2003.09.002.

[7] P. Heiselberg, M. Perino, Short-term airing by natural ventilation implication on IAQ and thermal comfort, Indoor Air 20 (2) (2010) 126-140. doi:10.1111/j.1600-0668.2009.00630.x.

[8] P. Carey, D. Etheridge, Direct wind tunnel modelling of natural ventilation for design purposes, Building Services Engineering Research and Technology 20 (3) (1999) 131-142. doi:10.1177/014362449902000305.

[9] G. Hunt, P. Linden, The fluid mechanics of natural ventilationdisplacement ventilation by buoyancy-driven flows assisted by wind, Building and Environment 34 (6) (1999) 707-720. doi:10.1016/S0360-1323(98)00053-5.

[10] G. R. Hunt, P. F. Linden, Steady-state flows in an enclosure ventilated by buoyancy forces assisted by wind, Journal of Fluid Mechanics 426 (2001) 355-386. doi:10.1017/S0022112000002470.

[11] Y. Li, A. Delsante, Natural ventilation induced by combined wind and thermal forces, Building and Environment 36 (1) (2001) 59-71. doi:10. 1016/S0360-1323(99) 00070-0.

[12] M. J. Cook, Y. Ji, G. R. Hunt, CFD Modelling of Natural Ventilation: Combined Wind and Buoyancy Forces, International Journal of Ventilation 1 (3) (2003) 169-179. doi:10.1080/14733315.2003.11683632.

[13] G. R. Hunt, P. F. Linden, Displacement and mixing ventilation driven by opposing wind and buoyancy, Journal of Fluid Mechanics 527 (2005) 27-55. doi:10.1017/S0022112004002575. 
[14] B. Lishman, A. W. Woods, On transitions in natural ventilation flow driven by changes in the wind, Building and Environment 44 (4) (2009) 666-673. doi:10.1016/j.buildenv. 2008.05.012.

[15] J. Yuan, L. R. Glicksman, Multiple steady states in combined buoyancy and wind driven natural ventilation: The conditions for multiple solutions and the critical point for initial conditions, Building and Environment 43 (1) (2008) 62-69. doi:10.1016/j.buildenv.2006.11.035.

[16] A. D. Stavridou, P. E. Prinos, Natural ventilation of buildings due to buoyancy assisted by wind: Investigating cross ventilation with computational and laboratory simulation, Building and Environment 66 (2013) 104-119. doi:10.1016/j.buildenv. 2013.04.011.

[17] T. S. Larsen, P. Heiselberg, Single-sided natural ventilation driven by wind pressure and temperature difference, Energy and Buildings 40 (6) (2008) 1031-1040. doi:10.1016/j.enbuild.2006.07.012.

[18] P. Heiselberg, K. Svidt, P. V. Nielsen, Characteristics of air flow from open windows, Building and Environment (2001) 11.

[19] L. J. Lo, A. Novoselac, Effect of indoor buoyancy flow on wind-driven cross ventilation, Building Simulation 6 (1) (2013) 69-79. doi:10.1007/ s12273-012-0094-3.

[20] B. H. Shaw, Heat and mass transfer by convection through large rectangular openings in vertical partitions, PhD Thesis, University of Glasgow (1976).

[21] M. Epstein, Buoyancy-Driven Exchange Flow Through Small Openings in Horizontal Partitions, Journal of Heat Transfer 110 (4a) (1988) 885-893. doi:10.1115/1.3250589.

[22] M. Epstein, M. A. Kenton, Combined Natural Convection and Forced Flow Through Small Openings in a Horizontal Partition, With Special Reference to Flows in Multicompartment Enclosures, Journal of Heat Transfer 111 (4) (1989) 980. doi:10.1115/1.3250814.

[23] D. E. Mowbray, The use of schlieren and shadowgraph techniques in the study of flow patterns in density stratified liquids, Journal of Fluid Mechanics 27 (3) (1967) 595-608. doi:10.1017/S0022112067000564.

[24] C. Cenedese, S. B. Dalziel, Concentration and depth fields determined by the light transmitted through a dyed solution, in: Proceedings of the 8th International Symposium on Flow Visualization, Vol. 8, 1998, pp. 1-37. 
[25] D. Allgayer, G. Hunt, On the application of the light-attenuation technique as a tool for non-intrusive buoyancy measurements, Experimental Thermal and Fluid Science 38 (2012) 257-261. doi:10.1016/j.expthermflusci. 2011.10.009.

[26] J. L. Partridge, P. F. Linden, Validity of thermally-driven small-scale ventilated filling box models, Experiments in Fluids 54 (11). doi:10.1007/ s00348-013-1613-4.

[27] D. Etheridge, Natural Ventilation through Large Openings - Measurements at Model Scale and Envelope Flow Theory, International Journal of Ventilation 2 (4) (2004) 325-342. doi:10.1080/14733315.2004.11683676.

[28] J. E. Cermak, M. Poreh, J. A. Peterka, S. S. Ayad, Wind Tunnel Investigations of Natural Ventilation, Journal of Transportation Engineering 110 (1) (1984) 67-79. doi:10.1061/(ASCE) 0733-947X (1984)110:1(67).

[29] Y.-H. Chiu, D. Etheridge, External flow effects on the discharge coefficients of two types of ventilation opening, Journal of Wind Engineering and Industrial Aerodynamics 95 (4) (2007) 225-252. doi:10.1016/j.jweia.2006. 06.013.

[30] D. E. Kiel, Buoyancy driven counterflow and interfacial mixing., Ph.D., University of Cambridge (1991).

[31] D. Wilson, D. Kiel, Gravity driven counterflow through an open door in a sealed room, Building and Environment 25 (4) (1990) 379-388. doi: 10.1016/0360-1323(90)90012-G.

[32] A. J. Ward-Smith, Internal Fluid Flow-The fluid dynamics of flow in pipes and ducts, Nasa Sti/recon Technical Report A 81.

[33] F. Flourentzou, J. Van der Maas, C. A. Roulet, Natural ventilation for passive cooling: measurement of discharge coefficients, Energy and Buildings 27 (3) (1998) 283-292. doi:10.1016/S0378-7788(97)00043-1.

[34] D. Etheridge, M. Sandberg, Building ventilation: theory and measurement, Vol. 50, John Wiley \& Sons Chichester, UK, 1996.

[35] S. Dalziel, G. Lane-Serff, The hydraulics of doorway exchange flows, Building and Environment 26 (2) (1991) 121-135. doi:10.1016/0360-1323(91) 90019-8.

[36] H. H. Schneider, Laboratory experiments to simulate the jet-induced erosion of pycnoclines in lakes, Proc. 2nd Int. Sym. Stratified Flows, Trondheim, Norwegian Institute of Technology. 
[37] W. D. Baines, Entrainment by a plume or jet at a density interface, Journal of Fluid Mechanics 68 (2) (1975) 309-320. doi:10.1017/ S0022112075000821.

[38] J. Herault, G. Facchini, M. Le Bars, Erosion of a sharp density interface by a turbulent jet at moderate Froude and Reynolds numbers, Journal of Fluid Mechanics 838 (2018) 631-657. doi:10.1017/jfm.2017.891.

[39] A. B. Shrinivas, G. R. Hunt, Confined turbulent entrainment across density interfaces, Journal of Fluid Mechanics 779 (2015) 116-143. doi:10.1017/ jfm.2015.366.

[40] T. L. Bergman, F. P. Incropera, D. P. DeWitt, A. S. Lavine, Fundamentals of heat and mass transfer, John Wiley \& Sons, 2011. 